\title{
SET MEMBERSHIP ESTIMATION OF PARAMETERS AND VARIABLES IN DYNAMIC NETWORKS BY RECURSIVE ALGORITHMS WITH A MOVING MEASUREMENT WINDOW
}

\author{
KAZIMIERZ DUZINKIEWICZ \\ Department of Automatic Control, Gdańsk University of Technology \\ ul. G. Narutowicza 11/12, 80-952 Gdańsk, Poland \\ e-mail: kduzin@ely.pg.gda.pl
}

\begin{abstract}
The paper considers a set membership joint estimation of variables and parameters in complex dynamic networks based on parametric uncertain models and limited hard measurements. A recursive estimation algorithm with a moving measurement window is derived that is suitable for on-line network monitoring. The window allows stabilising the classic recursive estimation algorithm and significantly improves estimate tightness. The estimator is validated on a case study regarding a water distribution network. Tight set estimates of unmeasured pipe flows, nodal heads, tank level and pipe resistances are obtained.
\end{abstract}

Keywords: set membership estimation, uncertainty, complexity, networks, water distribution

\section{Introduction}

A joint estimation of variables and model parameters is a routine activity that is carried out on-line during network operation. The estimation of heads, flow rates, tank level, chlorine concentrations, pipe parameters or chlorine reaction rates across drinking water distribution network (Brdyś and Chen, 1996; Brdyś and Lisiak, 1999; Brdyś et al., 2001; Duzinkiewicz, 2005), and the estimation of biological state and model parameters for an integrated wastewater system (Rutkowski et al., 2004) can serve as examples. It is crucial to properly integrate a-priori knowledge including mathematical models with measurement information provided by hard sensors in order to obtain robust and quality estimates. An uncertainty exists due to modelling errors, measurement noise and disturbance inputs. In the paper, in order to obtain robust estimates with a guaranteed estimation error and, at the same time, to reduce the modelling effort which is necessarily due to a network's complexity, a set membership model of uncertainty is employed (Chang et al., 2004; Duzinkiewicz, 2005; Milanese et al., 1996; Schweppe, 1978; Walter and Pronzato, 1997). For the linear case, the solution set is a convex polyhedron, which can be very complicated, and other simple-shaped forms, such as ellipsoids or parallelotopes, have been used to give an enclosure of the exact solution set (Milanese et al., 1996; Walter and Pronzato, 1997).

When a model is nonlinear, the previous algorithms are no longer relevant (Raïssi et al., 2004). In the paper, the outer approximation is used, defined by taking orthogonal projections of the exact set. The estimates are sets bounding uncertain parameters and tubes bounding uncertain variable trajectories. Point estimates can be selected from the set estimates depending on their future usage. Regardless of the selection, the estimation error can be always assessed in a guaranteed manner. The Chebyshev centres of the sets serve as point estimates that minimise the estimation error in the worst case (Milanese et al., 1996). In the paper, recursive algorithms for set membership estimation are derived. A moving measurement window is introduced in order to stabilize the estimates and also to compromise between the computational effort and estimate tightness.

It should be pointed out that the set bounded model of uncertainty is used in the paper in order to overcome difficulties in obtaining statistical information needed by a probabilistic model of uncertainty for estimation purposes. Moreover, key statistical assumptions are often not met in complex networks e.g., the whiteness of noise due to the modelling error. The "statistically optimal" estimates would not be then of the required quality. In addition, even if the network model is linear in parameters, it is rarely linear in variables that are estimated jointly. This creates well-known difficulties in applying probabilistic models of uncertainty. However, statistical concepts such as consistency, structure selection, etc. can also be applied to set membership estimation and similar results can be achieved (Veres and Norton, 1991). The paper does not focus on theoretical considerations with regard to these is- 
sues. Instead, a geometrical advantage of the set bounded approach is utilised in order to approximate complicated topological shapes of the set estimates, and their useful tightness is demonstrated by the application to an example regarding a water distribution network.

\section{Information in the Estimation Problem}

We shall consider a dynamic network that is composed of interconnected static and dynamic elements. The element variables and model parameters are related by equalities to obtain the element mathematical model. For a dynamic element, we shall distinguish between the state variables and the remaining non-state variables. The nonstate variables are composed of network inputs, outputs and intermediate variables. The other variables connect the elements. It is a common property of static network element models that all variables are linked in an implicit manner through model equalities. Hence, for a network as a whole, we shall distinguish between state variables $s$, non-state variables $\boldsymbol{y}$ and external inputs (controls and disturbances) $\boldsymbol{u}$. As all three types of variables are to be estimated (controls due to actuator errors), the composed vector $\boldsymbol{x}$ of the estimated variables is introduced as

$$
\boldsymbol{x}=\left[\boldsymbol{s}^{T}, \boldsymbol{u}^{T}, \boldsymbol{y}^{T}\right]^{T}, \boldsymbol{x} \in \mathbb{R}^{n_{x}}, \boldsymbol{u} \in \mathbb{R}^{n_{u}}, \boldsymbol{y} \in \mathbb{R}^{n_{y}} .
$$

Let us denote by $\mathcal{F}$ the operator representing the algebraic equations modelling the static part of the network. Hence, this static part of the network model can be written as

$$
\mathcal{F}(\boldsymbol{x})=\mathbf{0} \text {. }
$$

The operator $\mathcal{F}$ is not exactly known and only its approximate model $\mathcal{F}^{M}$ is available. The following holds:

$$
\mathbf{0}=\mathcal{F}(\boldsymbol{x})=\mathcal{F}^{M}(\boldsymbol{x}, \boldsymbol{\gamma})+\boldsymbol{e}_{s}^{M}(\boldsymbol{x}, \boldsymbol{\gamma}),
$$

where $\gamma \in \mathbb{R}^{n_{\gamma}}$ is the model parameter vector and $\boldsymbol{e}_{s}^{M}(\cdot)$ describes the modelling error of the static part of the model.

It is assumed that the upper and lower bounds on the modelling error, $e_{s}^{M, u}$ and $e_{s}^{M, l}$, respectively, are known, so that

$$
\boldsymbol{e}_{s}^{M, l} \leq \boldsymbol{e}_{s}^{M}(\boldsymbol{x}, \gamma) \leq \boldsymbol{e}_{s}^{M, u}
$$

The static network model used via (2)-(4) enables us to bound the sets of possible variable and parameter values to the ones that satisfy the inequalities

$$
-e_{s}^{M, u} \leq \mathcal{F}^{M}(\boldsymbol{x}, \gamma) \leq-\boldsymbol{e}_{s}^{M, l} .
$$

The relationship (5) can be written for the successive time steps $j=1,2, \ldots$ The inequality (5) with $\boldsymbol{x}(j), j=$ $1,2, \ldots$, bounds the variables and parameters at the time step $j$. By adding to this inequality known pointwise $a$ priori bounds on the estimated variables and parameters $\boldsymbol{x}^{\min }(j) \leq \boldsymbol{x}(j) \leq \boldsymbol{x}^{\max }(j)$ and $\boldsymbol{\gamma}^{\min } \leq \boldsymbol{\gamma} \leq \boldsymbol{\gamma}^{\max }$, static a-priori knowledge at the time step $j$ is obtained that can be shortly written as

$$
\mathcal{M}_{s}(\boldsymbol{x}(j), \gamma) \leq \mathbf{0}
$$

where $\mathcal{M}_{\int}$ is a suitably defined operator. Hence, the operator $\mathcal{M}_{\int}$ consists of the network static model inequalities and the $a$-priori bounds on the variables and parameters.

The network dynamics are composed of the element dynamics linked by intermediate variables as

$$
\boldsymbol{s}(j+1)=f(\boldsymbol{x}(j), j), \quad j=1,2, \ldots
$$

As previously, an approximate model $f^{M}(\cdot)$ of $f(\cdot)$ together with upper and lower bounds on the modelling error of the dynamic part of the model $\boldsymbol{e}_{d}^{M}(\boldsymbol{x}, \gamma, j)$, $\boldsymbol{e}_{d}^{M, u}(j)$ and $\boldsymbol{e}_{d}^{M, l}(j)$, respectively, is only available. The inequalities can then be written that robustly bound the estimated quantities at the time step $j$ as

$$
\boldsymbol{e}_{d}^{M, l}(j) \leq \boldsymbol{s}(j+1)-f^{M}(\boldsymbol{x}(j), \gamma, j) \leq \boldsymbol{e}_{d}^{M, u}(j),
$$

$j=1,2, \ldots$, yielding the dynamic a-priori knowledge at the time step $j$.

The models are assumed to be parametric, as opposed to point-parametric models (Chang et al., 2004). This means that there exists a constant parameter vector $\gamma^{*}$ and the modelling error mappings $e_{s}^{M, *}(\cdot)$ and $e_{d}^{M, *}(\cdot)$ such that for any external inputs from an admissible set, the following holds over the control horizon:

$$
\begin{aligned}
\mathcal{F}\left(\boldsymbol{x}^{*}(j)\right) & =\mathcal{F}^{M}\left(\boldsymbol{x}^{*}(j), \boldsymbol{\gamma}^{*}\right)+\boldsymbol{e}_{s}^{M}\left(\boldsymbol{x}^{*}(j), \boldsymbol{\gamma}^{*}\right) \\
f\left(\boldsymbol{x}^{*}(j), j\right) & =f^{M}\left(\boldsymbol{x}^{*}(j), \boldsymbol{\gamma}^{*}, j\right)+\boldsymbol{e}_{d}^{M}\left(\boldsymbol{x}^{*}(j), \boldsymbol{\gamma}^{*}, j\right), \\
\boldsymbol{e}_{s}^{M, l} & \leq \boldsymbol{e}_{s}^{M}\left(\boldsymbol{x}^{*}(j), \boldsymbol{\gamma}^{*}\right) \leq \boldsymbol{e}_{s}^{M, u} \\
\boldsymbol{e}_{d}^{M, l} & \leq \boldsymbol{e}_{d}^{M}\left(\boldsymbol{x}^{*}(j), \boldsymbol{\gamma}^{*}, j\right) \leq \boldsymbol{e}_{d}^{M, u}
\end{aligned}
$$

$j=1,2, \ldots$, where $\boldsymbol{x}^{*}(j)$ is the network variable trajectory over the control horizon. The vector $\gamma^{*}$ will be further called the real parameter vector.

Only a small part of the variable set is directly measured. Let $z$ denote the vector of the measured variables. Clearly, $\boldsymbol{z} \subset \boldsymbol{x}$. The measurements are taken at the discrete time instants $j$ corresponding to the time steps of the network dynamic model (7). The measurement set available at the time stage $k$ is

$$
\boldsymbol{Z}^{P}(k)=\left[\boldsymbol{z}^{P}(1)^{T}, \ldots, \boldsymbol{z}^{P}(j)^{T}, \ldots, \boldsymbol{z}^{P}(k)^{T}\right]^{T}
$$

where the superscript ' $P$ ' denotes hard sensor measurements of the network variables gathered over $j=\overline{1, k}$.

The measurements are contaminated by the noise $\boldsymbol{e}^{P}(j)$ and $\boldsymbol{z}^{P}(j)=\boldsymbol{z}(j)+\boldsymbol{e}^{P}(j), j=\overline{1, k}$. The noise 
is lower and upper bounded with the known $L_{\infty}$ bounds $\boldsymbol{e}^{P, l}(j)$ and $\boldsymbol{e}^{P, u}(j)$, respectively. Hence, the following holds for $j \in \overline{1, k}$ :

$$
\boldsymbol{z}^{P}(j)-\boldsymbol{e}^{P, u}(j) \leq \boldsymbol{z}(j) \leq \boldsymbol{z}^{P}(j)-\boldsymbol{e}^{P, l}(j) .
$$

The inequalities (11) over the time period $\Xi_{k}=\overline{1, k}$ constitute the overall information about the variables and parameters available at the time instant $k$ from the measurements gathered till $k$. We shall call it the measurement information at the time step $k$. Let $\boldsymbol{X}(k)$ be a vector of the trajectories of the estimated variables over $\Xi_{k}=\overline{1, k}$. That is,

$$
\boldsymbol{X}(k)=\left[\boldsymbol{x}(1)^{T}, \ldots, \boldsymbol{x}(j)^{T}, \ldots, \boldsymbol{x}(k)^{T}\right]^{T} .
$$

The inequalities (11) can now be briefly written as

$$
\mathcal{P}(k)(\boldsymbol{X}(k)) \leq \mathbf{0},
$$

where $\mathcal{P}(\cdot)$ is a suitably defined operator. We shall also introduce an operator $p(k)$ to define the measurement inequality $p(k)(\boldsymbol{x}(k)) \leq \mathbf{0}$ only at $k$ :

$$
\boldsymbol{z}^{P}(k)-\boldsymbol{e}^{P, u}(k) \leq \boldsymbol{z}(k) \leq \boldsymbol{z}^{P}(k)-\boldsymbol{e}^{P, l}(k) .
$$

\section{Batch Estimation Algorithms}

\subsection{Static Joint Batch Estimation of Variables and}

Parameters. At the time instant $k$, let us consider all estimated variable trajectories $\boldsymbol{X}(k)$ and all parameter values $\gamma$ consistent with the static a-priori knowledge and the measurements over the time period $\Xi_{k}=\overline{1, k}$. Let $\boldsymbol{\Phi}(k)$ denote the set of all these consistent variable trajectories and parameter values. Hence, the set $\boldsymbol{\Phi}(k)$ can be defined in the following way:

$$
\begin{aligned}
\boldsymbol{\Phi}(k)=\{ & (\boldsymbol{X}(k), \boldsymbol{\gamma}): \mathcal{M}_{s}(\boldsymbol{x}(j), \boldsymbol{\gamma}) \leq \mathbf{0}, j=\overline{1, k} \\
& \mathcal{P}(k)(\boldsymbol{X}(k)) \leq \mathbf{0}\} .
\end{aligned}
$$

If $(\boldsymbol{X}(k), \gamma) \in \boldsymbol{\Phi}(k)$, then, on the basis of the static a-priori information and measurement information available at the time instant $k$, we cannot preclude that the end point $\boldsymbol{x}(k)$ of the trajectory $\boldsymbol{X}(k)$ is equal to the real vector $\boldsymbol{x}^{*}(k)$ and the parameter vector $\boldsymbol{\gamma}$ is equal to the real parameter vector $\gamma^{*}$ estimated at the time instant $k$. Let $\boldsymbol{\Phi}_{\boldsymbol{x}(k), \boldsymbol{\gamma}}(k)$ denote the set of the end points of the variable trajectories $\boldsymbol{X}(k)$ and parameter values $\boldsymbol{\gamma}$ such that $(\boldsymbol{X}(k), \gamma) \in \boldsymbol{\Phi}(k)$. It is obvious that for the static a-priori information and measurement information available at the time instant $k$, the set $\boldsymbol{\Phi}_{\boldsymbol{x}(k), \boldsymbol{\gamma}}(k)$ is the smallest one that certainly contains the estimated pair $\left(\boldsymbol{x}^{*}(k), \boldsymbol{\gamma}^{*}\right)$. It should be noted that, in spite of estimating variables only at the time instant $k$, the static a-priori information at all time instants over the period $\Xi_{k}=\overline{1, k}$ has been taken into account. This is due to the time structure of the static batch estimation problem introduced to it by constant values of the parameters.

It is a very difficult numerical task to obtain exact bounds of the set $\boldsymbol{\Phi}_{\boldsymbol{x}(k), \boldsymbol{\gamma}}(k)$. Hence, the estimates of the variables $\boldsymbol{x}(k)$ and parameters $\boldsymbol{\gamma}$ are defined as orthogonal projections of $\boldsymbol{\Phi}_{\boldsymbol{x}(k), \boldsymbol{\gamma}}(k)$ on the subspaces of a variable $x_{i}(k)$ and parameter $\gamma_{i}$, the elements of the vector $\boldsymbol{x}(k)$ and $\gamma$, respectively. The intervals $\left[x_{i}^{\min }(k), x_{i}^{\max }(k)\right]$ and $\left[\gamma_{i}^{\min }(k), \gamma_{i}^{\max }(k)\right]$ are then obtained by bounding at $k$ the scalars $x_{i}^{*}(k)$ and $\gamma_{i}^{*}$. The following holds for each pair of the components $\left(x_{i}^{*}, \gamma_{i}^{*}\right)$ of the vector $\left(\boldsymbol{x}^{*}(k), \gamma^{*}\right)$ :

$$
\begin{gathered}
x_{i}^{\min }(k) \leq x_{i}^{*}(k) \leq x_{i}^{\max }(k), \\
\gamma_{i}^{\min }(k) \leq \gamma_{i}^{*} \leq \gamma_{i}^{\max }(k) .
\end{gathered}
$$

An orthotope built by the interval estimates (16) is only an outer approximation of the set $\boldsymbol{\Phi}_{\boldsymbol{x}(k), \boldsymbol{\gamma}}(k)$. It may happen that this approximation is conservative and contains many points not consistent with the information available at the time instant $k$. The centres of the interval estimates (16) are natural choices of robust point estimates as it may be possible to assess an upper error of these estimates. In other words, it will be possible to determine the guaranteed estimation error.

The interval estimate of the $i$-th components of the variable vector $\boldsymbol{x}(k)$ and the parameter vector $\gamma$ are determined by solving the following optimisation tasks:

$$
\begin{aligned}
& \min (\max )\left\{q_{o}(\boldsymbol{X}(k), \gamma)=x_{i}(k)\left(\text { or } \gamma_{i}\right)\right\} \\
& \text { subject to }(\boldsymbol{X}(k), \gamma) \in \mathbf{\Phi}(k),
\end{aligned}
$$

where $q_{0}$ is the objective function for determining the lower and upper bounds of the outer approximation set.

The application of static estimation to the dynamic network is practically possible when the changeability of system variables is small. Then the dynamic a-priori information (8) over the time period $\Xi_{k}=\overline{1, k}$ can be replaced by the static a-priori information in the form of the inequality

$$
\boldsymbol{e}_{d}^{M, l}(j) \leq \boldsymbol{s}-\boldsymbol{f}^{M}(\boldsymbol{x}(j), \boldsymbol{\gamma}, j) \leq \boldsymbol{e}_{d}^{M, u}(j),
$$

$j=\overline{1, k}$, where $s$ is a constant vector.

The inequalities (18) should be added to the inequalities set (15), and the resulting extended task of the static estimation should be solved.

\subsection{Dynamic Joint Batch Estimation of Variables and} Parameters. The static joint estimation of variables and parameters at the time instant $k$ did not utilise the dynamic a-priori information given by (8) over the time interval $\Xi_{k}=\overline{1, k}$. At the time instant $k$ we shall define a set $\boldsymbol{\Omega}(k)$ 
of all variable trajectories $\boldsymbol{X}(k)$ and parameters $\boldsymbol{\gamma}$ that are consistent with the measurement and a-priori information over the time period $\Xi_{k}=\overline{1, k}$ which is available at $k$. Hence, the following holds:

$$
\begin{aligned}
& \boldsymbol{\Omega}(k)=\{(\boldsymbol{X}(k), \boldsymbol{\gamma}, \boldsymbol{s}(k+1)): \\
& \mathcal{M}_{s}(\boldsymbol{x}(j), \gamma) \leq \mathbf{0}, \quad j=\overline{1, k} ; \\
& \boldsymbol{e}_{d}^{M, l}(j) \leq \boldsymbol{s}(j+1)-f^{M}(\boldsymbol{x}(j), \boldsymbol{\gamma}, j) \\
& \leq \boldsymbol{e}_{d}^{M, u}(j), \quad j=\overline{1, k} ; \\
&\mathcal{P}(k)(\boldsymbol{X}(k)) \leq \mathbf{0}\} .
\end{aligned}
$$

The state $s(k)$ is estimated as a component of the vector $\boldsymbol{x}(k)$. The state $\boldsymbol{s}(k+1)$ participates in the definition of the set $\boldsymbol{\Omega}(k)$ since the inequality (8) with $j=k$ contributes at $k$ to knowledge about $\boldsymbol{x}(k)$, and $\boldsymbol{s}(k+1)$ is present in (8). However, as opposed to $\boldsymbol{x}(k)$ and $\boldsymbol{\gamma}$, estimating $\boldsymbol{s}(k+1)$ at $k$ means predicting the state.

Let $(\boldsymbol{X}(k), \boldsymbol{\gamma}, \boldsymbol{s}(k+1)) \in \boldsymbol{\Omega}(k)$. Regarding the static, dynamic and measurement information available at $k$, the set

$$
\boldsymbol{\Omega}_{\boldsymbol{x}(k), \boldsymbol{\gamma}, \boldsymbol{s}(k+1)}(k) \triangleq\{(\boldsymbol{x}(k), \boldsymbol{\gamma}, \boldsymbol{s}(k+1)) \subset \boldsymbol{\Omega}(k)\}
$$

is the smallest set which is guaranteed to contain the estimated quantities $\left(\boldsymbol{x}^{*}(k), \boldsymbol{\gamma}^{*}, \boldsymbol{s}^{*}(k+1)\right)$. Unfortunately, as for the static estimation, a topological structure of this set is complicated even for linear networks. Hence, for practical reasons, we shall take its outer approximation that can be realistically determined as the set estimate at $k$. The outer approximation is defined by taking orthogonal projections of $\boldsymbol{\Omega}_{\boldsymbol{x}(k), \boldsymbol{\gamma}, \boldsymbol{s}(k+1)}(k)$ on the subspaces of $x_{i}(k), s_{i}(k+1)$ and $\gamma_{i}$. This results in producing the intervals $\left[x_{i}^{\min }(k), x_{i}^{\max }(k)\right],\left[s_{i}^{\min }(k+1), s_{i}^{\max }(k+1)\right]$ and $\left[\gamma_{i}^{\min }(k), \gamma_{i}^{\max }(k)\right]$ bounding $x_{i}^{*}(k), s_{i}^{*}(k+1)$ and $\gamma_{i}^{*}$ at $k$, respectively. This means that the following holds:

$$
\begin{aligned}
& x_{i}^{\min }(k) \leq x_{i}^{*}(k) \leq x_{i}^{\max }(k), \\
& \gamma_{i}^{\min }(k) \leq \gamma_{i}^{*} \leq \gamma_{i}^{\max }(k), \\
& s_{i}^{\min }(k+1) \leq s_{i}^{*}(k+1) \leq s_{i}^{\max }(k+1) .
\end{aligned}
$$

Let the sets $\boldsymbol{\Omega}_{\boldsymbol{x}(k)}(k), \boldsymbol{\Omega}_{\boldsymbol{s}(k+1)}(k)$ and $\boldsymbol{\Omega}_{\boldsymbol{\gamma}}(k)$ be outer approximations of the smallest set which are guaranteed to contain the estimated quantities $\boldsymbol{x}^{*}(k), \boldsymbol{\gamma}^{*}$, and $\boldsymbol{s}^{*}(k+1)$, respectively. The sets $\boldsymbol{\Omega}_{\boldsymbol{x}(k)}(k), \boldsymbol{\Omega}_{\boldsymbol{s}(k+1)}(k)$ and $\boldsymbol{\Omega}_{\gamma}(k)$ are defined by

$$
\begin{aligned}
\boldsymbol{\Omega}_{\boldsymbol{x}(k)}(k) \triangleq & {\left[x_{1}^{\min }(k), x_{1}^{\max }(k)\right] } \\
& \times \cdots \times\left[x_{i}^{\min }(k), x_{i}^{\max }(k)\right] \\
& \times \cdots \times\left[x_{n_{x}}^{\min }(k), x_{n_{x}}^{\max }(k)\right] \in \mathbb{R}^{n_{x}},
\end{aligned}
$$

$$
\begin{aligned}
\boldsymbol{\Omega}_{\gamma}(k) \triangleq & {\left[\gamma_{1}^{\min }(k), \gamma_{1}^{\max }(k)\right] } \\
& \times \cdots \times\left[\gamma_{i}^{\min }(k), \gamma_{i}^{\max }(k)\right] \\
& \times \cdots \times\left[\gamma_{n_{\gamma}}^{\min }(k), \gamma_{n_{\gamma}}^{\max }(k)\right] \in \mathbb{R}^{n_{\gamma}},
\end{aligned}
$$

$$
\begin{aligned}
\boldsymbol{\Omega}_{\boldsymbol{s}(k+1)}(k) \triangleq & {\left[s_{1}^{\min }(k+1), s_{1}^{\max }(k+1)\right] } \\
& \times \cdots \times\left[s_{i}^{\min }(k+1), s_{i}^{\max }(k+1)\right] \\
& \times \cdots \times \\
& {\left[s_{n_{s}}^{\min }(k+1), s_{n_{s}}^{\max }(k+1)\right] \in \mathbb{R}^{n_{s}} . }
\end{aligned}
$$

Clearly, the following holds:

$\boldsymbol{\Omega}_{\boldsymbol{x}(k), \boldsymbol{\gamma}, \boldsymbol{s}(k+1)}(k) \subset \boldsymbol{\Omega}_{\boldsymbol{x}(k)}(k) \times \boldsymbol{\Omega}_{\boldsymbol{\gamma}}(k) \times \boldsymbol{\Omega}_{\boldsymbol{s}(k+1)}(k)$.

The Cartesian products of the sets $\boldsymbol{\Omega}_{\boldsymbol{x}(k)}(k), \boldsymbol{\Omega}_{\boldsymbol{s}(k+1)}(k)$ and $\Omega_{\gamma}(k)$ constitute outer approximations of the set $\boldsymbol{\Omega}_{\boldsymbol{x}(k), \boldsymbol{\gamma}, \boldsymbol{s}(k+1)}(k)$ determined by solving the following optimisation tasks:

$$
\begin{gathered}
\min (\max )\left\{q_{0}(\boldsymbol{X}(k), \boldsymbol{\gamma}, \boldsymbol{s}(k+1))=x_{i}(k)\right. \\
\left.\left(\text { or } \gamma_{i} \text { or } s_{i}(k+1)\right)\right\} \\
\text { subject to }(\boldsymbol{X}(k), \boldsymbol{\gamma}, \boldsymbol{s}(k+1)) \in \boldsymbol{\Omega}(k),
\end{gathered}
$$

where $q_{0}$ is an objective function for determining the lower and upper bounds of the outer approximation set.

\section{Recursive Estimation Algorithms}

4.1. Dynamic Joint Recursive Estimation of Variables and Parameters with Standard Recursion. The formulation (19) and (24), called the batch estimation, is not suitable for on-line applications. We shall now derive a recursive formulation. Let us compare the sets $\boldsymbol{\Omega}(k)$ and $\boldsymbol{\Omega}(k-1)$, which constitute the information bases for the estimation at $k$ and $k-1$, respectively. The new information available at $k$ is the measurement information gathered at $k$. Moreover, at $k$, the new variables $\boldsymbol{x}(k)$ and $\boldsymbol{s}(k+1)$ are to be estimated. Accordingly, at the time instant $k$, the following information is added to the set $\boldsymbol{\Omega}(k)$ : 
the static a-priori information about variables and parameters at $k$ given by (6), the dynamic a-priori information about variables and parameters changeable at $k$ given by (8) and the measurement information given by (14). This new piece of information can be then expressed as

$$
\begin{gathered}
\boldsymbol{\omega}(k)=\{(\boldsymbol{x}(k), \boldsymbol{\gamma}, \boldsymbol{s}(k+1)): \\
\mathcal{M}_{s}(\boldsymbol{x}(k), \boldsymbol{\gamma}) \leq \mathbf{0}, p(k)(\boldsymbol{x}(k)) \leq \mathbf{0}, \\
\boldsymbol{e}_{d}^{M, l}(k) \leq \boldsymbol{s}(k+1)-f^{M}(\boldsymbol{x}(k), \boldsymbol{\gamma}, k) \\
\left.\leq \boldsymbol{e}_{d}^{M, u}(k)\right\} .
\end{gathered}
$$

The set $\boldsymbol{\Omega}(k)$ can now be written in the recursive form as

$$
\begin{aligned}
\boldsymbol{\Omega}(k)=\{ & (\boldsymbol{X}(k), \boldsymbol{\gamma}, \boldsymbol{s}(k+1)): \\
& (\boldsymbol{X}(k-1), \boldsymbol{\gamma}, \boldsymbol{s}(k)) \in \boldsymbol{\Omega}(k-1), \\
& (\boldsymbol{x}(k), \boldsymbol{\gamma}, \boldsymbol{s}(k+1)) \in \boldsymbol{\omega}(k)\} .
\end{aligned}
$$

Comparing the sets $\boldsymbol{\Omega}(k-1)$ and $\boldsymbol{\omega}(k)$ in (26), it can be stated that the parameters $\gamma$ and variables $s(k)$ occur in both these sets as a component of the vector $\boldsymbol{x}(k)$. Knowledge about the parameter vector $\gamma$ and state vector $\boldsymbol{s}(k)$ resulting from the estimation at the time instant $k-1$ is represented by the sets $\boldsymbol{\Omega}_{\boldsymbol{\gamma}}(k-1)$ and $\boldsymbol{\Omega}_{\boldsymbol{s}(k)}(k-1)$. According to (23), we have

$$
\begin{aligned}
\boldsymbol{\Omega}_{\boldsymbol{x}(k-1), \boldsymbol{\gamma}, \boldsymbol{s}(k)}(k-1) \subset \boldsymbol{\Omega}_{\boldsymbol{x}(k-1)}(k-1) & \\
& \times \boldsymbol{\Omega}_{\boldsymbol{\gamma}}(k-1) \times \boldsymbol{\Omega}_{\boldsymbol{s}(k)}(k-1) .
\end{aligned}
$$

Hence, the sets $\boldsymbol{\Omega}_{\boldsymbol{\gamma}}(k-1)$ and $\boldsymbol{\Omega}_{\boldsymbol{s}(k)}(k-1)$ are only outer approximations of the sets representing information about $\gamma$ and $s(k)$ that are contained in the set $\Omega(k-1)$. At the time instant $k$, the recursive form of the estimation algorithm generates the interval estimates by solving the following optimisation tasks:

$$
\begin{aligned}
& \min (\max )\left\{q_{0}(\boldsymbol{X}(k), \boldsymbol{\gamma}, \boldsymbol{s}(k+1))=x_{i}(k)\right. \\
& \left.\left(\text { or } \gamma_{i} \text { or } s_{i}(k+1)\right)\right\} \\
& \text { subject to }(\boldsymbol{x}(k), \boldsymbol{\gamma}, \boldsymbol{s}(k+1)) \in \boldsymbol{\omega}(k) \text {, } \\
& \gamma \in \boldsymbol{\Omega}_{\boldsymbol{\gamma}}(k-1) \text {, } \\
& \boldsymbol{s}(k) \in \boldsymbol{\Omega}_{\boldsymbol{s}(k)}(k-1),
\end{aligned}
$$

where the set estimates $\boldsymbol{\Omega}_{\boldsymbol{\gamma}}(k-1)$ and $\boldsymbol{\Omega}_{\boldsymbol{s}(k)}(k-1)$ are known from the estimation at $k-1$.

The estimation at $k=1$ uses the initial state estimates $s_{0} \in S_{0}$ known a priori at the time instant $k=0$. Hence $\boldsymbol{\Omega}_{\boldsymbol{s}(1)}(0)=S_{0}$. Similarly, $\boldsymbol{\Omega}_{\boldsymbol{\gamma}}(0)=\boldsymbol{\Gamma}_{0}$, where $\Gamma_{0}$ is a-priori knowledge about a set bound $\gamma$. It should be noted that the state prediction $s(k)$ obtained at $k-1$ is improved by the estimation algorithm at $k$ as a result of using the new measurement information gathered at $k$.

Notice that due to (23) and (27), a conservatism exists in the estimates defined by (28) when compared with the batch estimates, and this is the price to be paid for the recursive structure of the estimation algorithm. This conservatism may produce an unstable process of generating points that are not consistent with the available information.

\subsection{Dynamic Joint Recursive Estimation of Variables} and Parameters with a Moving Measurement Window. The stability of the recursive estimation algorithm may be regained by directly introducing a number of past measurements into the estimation task that is performed at $k$. They form a measurement window with $L$ past measurements. In the recursive algorithm (28), $L=0$. Since the measurement information is coupled with the a-priori information through the model (see (19)), the overall information that is utilised for the estimation at $k$ can be decomposed into the information used to perform the estimation at $k-L-1$ that means $\Omega(k-L-1)$ and the new information over the window $\boldsymbol{\omega}(k-L, k)$. Hence,

$$
\begin{aligned}
& \boldsymbol{\omega}(k-L, k)=\{(\boldsymbol{X}(k-L, k), \boldsymbol{\gamma}, \boldsymbol{s}(k+1)): \\
& \mathcal{M}_{s}(\boldsymbol{x}(j), \boldsymbol{\gamma}) \leq \mathbf{0}, \quad j=\overline{k-L, k} ; \\
& \mathcal{P}(k-L, k)(\boldsymbol{X}(k-L, k)) \leq \mathbf{0} ; \\
& \boldsymbol{e}_{d}^{M, l}(j) \leq \boldsymbol{s}(j+1)-f^{M}(\boldsymbol{x}(j), \boldsymbol{\gamma}, j) \\
&\left.\leq \boldsymbol{e}_{d}^{M, u}(j), \quad j=\overline{k-L, k}\right\}, \quad \text { (29) }
\end{aligned}
$$

where $\boldsymbol{X}(k-L, k)$ is the set of all variable trajectories over the time period $\Xi_{-L}=\overline{k-L, k}$.

Hence, the information base $\boldsymbol{\Omega}(k)$ for the estimation performed at $k$ can be written in the form equivalent to (19) and (26) as follows:

$$
\begin{aligned}
\boldsymbol{\Omega}(k)=\{ & (\boldsymbol{X}(k), \boldsymbol{\gamma}, \boldsymbol{s}(k+1)): \\
& (\boldsymbol{X}(k-L-1), \boldsymbol{\gamma}, \boldsymbol{s}(k-L)) \in \boldsymbol{\Omega}(k-L-1) \\
& (\boldsymbol{X}(k-L, k), \boldsymbol{\gamma}, \boldsymbol{s}(k+1)) \in \boldsymbol{\omega}(k-L, k)\},
\end{aligned}
$$

where the set $\Omega(k-L-1)$ is the information base for the estimation task that is solved at $k-L-1$.

Notice that knowledge about the parameter $\gamma$ and state $s(k-L)$ gathered as a result of the estimation performed at $k-L-1$ and based on the set $\Omega(k-L-1)$ is expressed by the sets $\boldsymbol{\Omega}_{\boldsymbol{x}(k-L-1)}(k-L-1)$, $\boldsymbol{\Omega}_{\boldsymbol{\gamma}}(k-L-1)$ and $\boldsymbol{\Omega}_{\boldsymbol{s}(k-L)}(k-L-1)$. The first set 
has no influence on the estimation results at $k$. Hence, the recursive algorithm with a moving measurement window of the length $L$ can be formulated at $k$ as follows:

$$
\begin{aligned}
& \min (\max )\left\{q_{0}(\boldsymbol{X}(k-L, k), \boldsymbol{\gamma}, \boldsymbol{s}(k+1))=x_{i}(k)\right. \\
& \left.\left(\text { or } \gamma_{i} \text { or } s_{i}(k+1)\right)\right\} \\
& \text { subject to }(\boldsymbol{X}(k-L, k), \boldsymbol{\gamma}, \boldsymbol{s}(k+1)) \in \boldsymbol{\omega}(k-L, k) \text {, } \\
& \gamma \in \boldsymbol{\Omega}_{\gamma}(k-L-1) \text {, } \\
& \boldsymbol{s}(k-L) \in \boldsymbol{\Omega}_{\boldsymbol{s}(k-L)}(k-L-1),
\end{aligned}
$$

where the sets $\boldsymbol{\Omega}_{\boldsymbol{s}(k-L)}(k-L-1)$ and $\boldsymbol{\Omega}_{\boldsymbol{\gamma}}(k-L-1)$ come from the estimation performed at $k-L-1$.

Let us notice that the original information inequalities, but not their external approximations, are processed over the window. This has the above-mentioned stabilising impact on the estimates. The prediction $\boldsymbol{\Omega}_{\boldsymbol{s}(k+1)}(k)$ of the state $s(k+1)$ determined at $k$ will be used during the estimation carried out at $k+L+1$ as an estimate of the initial condition. At $k+1$, the window moves ahead by one time step and the state $s(k+1)$ will enter the window. Hence, the set $\boldsymbol{\Omega}_{\boldsymbol{s}(k+1)}(k)$ can be used at $k+1$ as an additional constraint bounding $s(k)$. Introducing the constraint

$$
\boldsymbol{s}(k) \in \boldsymbol{\Omega}_{\boldsymbol{s}(k))}(k-1)
$$

into the constraint set of (31) does this.

Remaining within the window for some time creates an opportunity for smoothing the past estimates based on information about the new measurements. However, this requires solving additional optimisation tasks. The smoothing, although expensive, may vastly improve the tightness of estimates. We shall limit ourselves to exploiting only one possibility. During the estimation performed at $k$, the state $s(k-L+1)$ becomes an initial state condition for the estimation to be carried out at $k+1$. It is therefore beneficial for the estimation performance at $k+1$ to estimate this state also at $k$. By including this and (32) into (31), the following estimation algorithm is obtained:

$$
\begin{aligned}
\min (\max )\{ & q_{0}(\boldsymbol{X}(k-L, k), \boldsymbol{\gamma}, \boldsymbol{s}(k+1))=x_{i}(k) \\
& \text { or } \left.s_{i}(k-L+1) \text { or } \boldsymbol{\gamma}_{i} \text { or } \boldsymbol{s}_{i}(k+1)\right\}
\end{aligned}
$$

subject to $(\boldsymbol{X}(k-L, k), \boldsymbol{\gamma}, \boldsymbol{s}(k+1)) \in \boldsymbol{\omega}(k-L, k)$,

$$
\begin{aligned}
& \boldsymbol{\gamma} \in \boldsymbol{\Omega}_{\boldsymbol{\gamma}}(k-L-1), \\
& \boldsymbol{s}(k-L) \in \boldsymbol{\Omega}_{\boldsymbol{s}(k-L)}(k-L-1), \\
& \boldsymbol{s}(k) \in \boldsymbol{\Omega}_{\boldsymbol{s}(k)}(k-1) .
\end{aligned}
$$

We have then $\boldsymbol{\Omega}_{\boldsymbol{s}(k-L)}(k-L-1)=\boldsymbol{\Omega}_{\boldsymbol{s}(k)}(k-1)$ if $L=0$. A routine state prediction is then sufficient in (33). Hence, the beneficial smoothing of the initial condition can be achieved only when the moving measurement window is applied to the estimation algorithm.

\section{Simulation Results for a Case Study}

A case study regarding the drinking water network of Fig. 1 will be investigated. The operators $\mathcal{F}^{M}(\cdot)$ and $f^{M}(\cdot)$ can be defined by the following discrete equations for the current time instant $k$ :

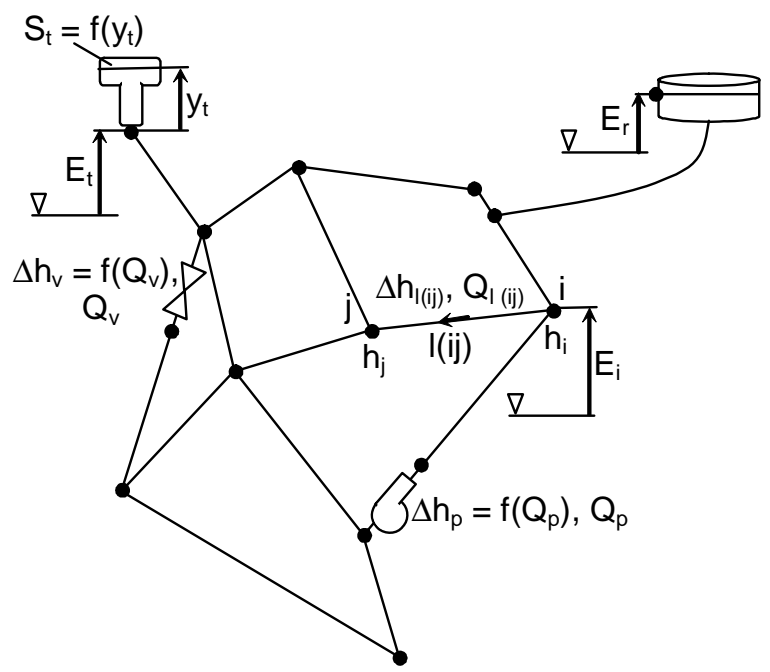

Quantities:

$\mathrm{Q}$ - pipe flow

E - reservoir or tank elevation

$\mathrm{y}$ - water level in a tank

$\mathrm{h}$ - head at a junction node

$\Delta \mathrm{h}$ - change of the head along a link

$\mathrm{S}$ - cross area of a tank

$$
\begin{aligned}
& \text { Subscripts: } \\
& \mathrm{t} \text { - tank } \\
& \mathrm{r} \text { - reservoir } \\
& \mathrm{v}-\text { valve } \\
& \mathrm{p} \text { - pump } \\
& \mathrm{i}, \mathrm{j} \text { - node number } \\
& \mathrm{l} \text { - pipe number }
\end{aligned}
$$

Fig. 1. Drinking water network.

1. Flow continuity law:

$$
\forall i: \sum_{l \in P_{i}^{+}} Q_{l(j i)}(k)-\sum_{l \in P_{i}^{-}} Q_{l(i j)}(k)=d_{i}(k),
$$

where $P_{i}^{+}$is the inflow (flow toward the junction node) of the $i$-th node and $P_{i}^{-}$stands for the outflow (flow from the junction node) of the $i$-th node, and $d_{i}$ signifies the water demand at the $i$-th node.

2. Generalized link equation:

$$
\forall l(i j): h_{i}(k)-h_{j}(k)=\Delta h_{l(i j)}(k),
$$

where the water head $h$ or its change $\Delta h$ are determined according to the type of node or the type of link.

For a tank node, we have

$$
\begin{aligned}
h_{t}(k) & =E_{t}+y_{t}(k), \\
y_{t}(k+1) & =y_{t}(k)+\frac{1}{S_{t}} Q_{t(i t)}(k) \Delta t,
\end{aligned}
$$

where $\Delta t$ is the a hydraulic time step. 
For a reservoir node, we get

$$
h_{r}(k)=E_{r}(k) .
$$

For a pipe link (the Hazen-Williams formula),

$$
\Delta h_{l(i j)}(k)=R_{l} Q_{l(i j)}^{1.852}(k),
$$

where $R$ is the resistance of the pipe.

For a pump link, we have

$$
\Delta h_{p(i j)}(k)=A_{p} Q_{p(i j)}^{2}(k)+B_{p} Q_{p(i j)}(k)+h_{u, p},
$$

where $A, B$ and $h_{u}$ are parameters of the pump curve.

For a valve link

$$
\Delta h_{v(i j)}(k)=R_{v} Q_{v(i j)}^{2}(k),
$$

where $R$ is the resistance of a valve.

The relations (34), (35) and (38)-(41) constitute the static part of the network model defining the operator $\mathcal{F}^{M}(\cdot)$, cf. (3). The tank is a dynamic element that is modelled by the relations (36) and (37) defining the operator $f^{M}(\cdot)$, cf. (8).

The paper considers a case study of a distribution system of a medium size city of Lębork in Poland. The network variables to be monitored are flows through the pipes, pressures at the network, tank nodes and water demands. The pipe resistances are model parameters. The measurements are typically limited due to the cost of sensor maintenance, and unmeasured quantities need to be estimated by using the model and hard measurements. A skeleton of the system considered is shown in Fig. 2.

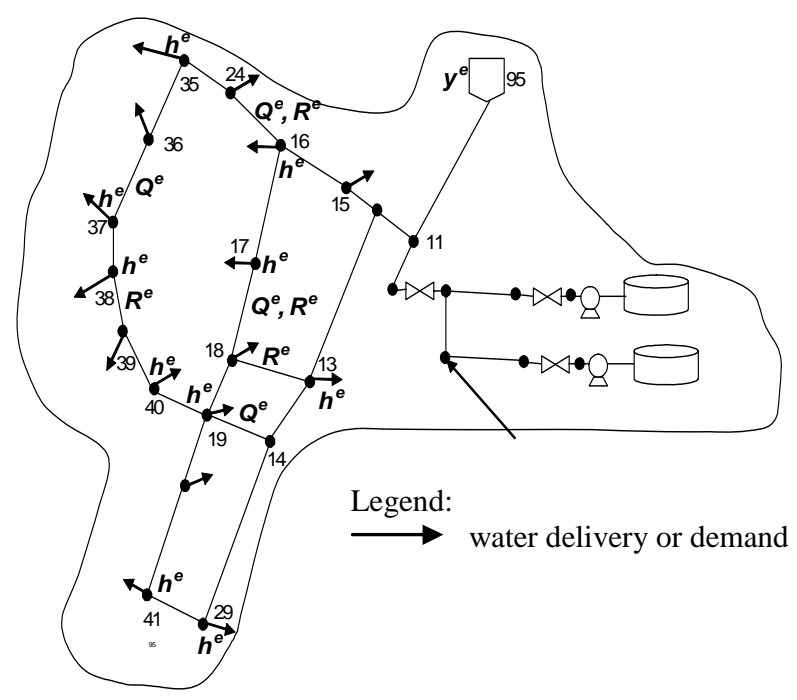

Fig. 2. Lębork case study network.

The distribution system consists of 19 demand nodes, 24 pipes and one tank. In Fig. 2, the estimated variables are marked $h^{e}$ for the nodal heads and $Q^{e}$ for the pipe flows, while the parameters are marked by $R^{e}$. All other nodal demands, pipe flows and nodal heads are measured. The measurement error bounds are: flow $\pm 3 \%$, nodal head $\pm 3 \%$, pipe resistance $\pm 10 \%$ and demand $\pm 10 \%$. The computations were performed on an IBM PC equipped with a PIII $933 \mathrm{MHz}$ processor, using the GAMS v.19.6 package. The solver used in solving the optimisation tasks was MIP CPLEX.

The nonlinear non-convex optimisation tasks of the estimation problem (33) were first converted into an approximated piecewise linear form and then solved by applying a mixed integer linear solver (Brdyś et al., 2001; Duzinkiewicz, 2005) to produce the desired global optima. The linearisation error was handled by introducing the error into the formulation of the estimation problem as a modelling error.

The piecewise linearisation introduces additional variables, and the number of these new variables rapidly increases if the original variable domains (ranges) increase. In order to maintain the linearisation ranges as small as possible, flow balance equations are utilised to predict the ranges for the next time instant (Brdys et al., 2001). This is called a dynamic grid allocation, which is crucial for on-line applications.

A simple recursive algorithm with $L=0$ and recursive estimation with a moving measurement window of the window length $L=1$ and $L=2$ were applied to estimate the pipe flows, nodal heads and pipe resistances. The results for $L=0$ and $L=2$ are shown in Figs. 3-12. Stable and tight estimating bounds were obtained that are illustrated by upper and lower bounding envelopes. The true trajectories of the estimated flows and heads lie within the envelopes and the resistance bounds get closer.

Differences in recursive estimation with the moving measurement window method for various window lengths can be easily seen in the resistance, tank level and pipe flow estimation processes. For example, according to the a-priori knowledge about the resistance R17_18, its value was between 0 and 0.7 . After 24 hours of recursive estimation carried out during the normal system operation and, hence, without applying specially designed identification inputs, the uncertainty in the resistance value was reduced to the interval $[0.085,0.18]$ for the window length $L=0,[0.09,0.18]$ for $L=1$ and $[0.13,0.17]$ for $L=2$.

One can also notice some distortion in the estimation results, especially for nodal heads. They appear because only a suboptimal value of the integer component of the optimized variables was found in order to improve the computational efficiency of the algorithm. Therefore, the resulting estimates constitute a compromise between the quality of the estimates and computational performance. As has been expected, better estimates are produced for $L=2$. Nevertheless, an approach to the selection of the appropriate values for windows length $L$ needs further investigations. 


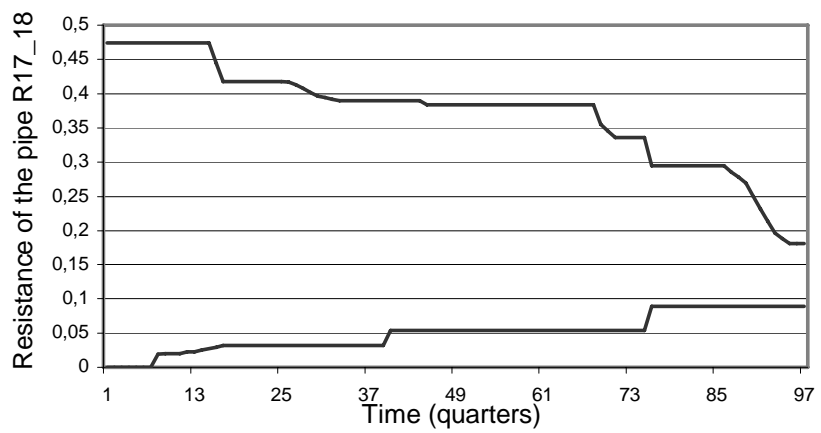

Fig. 3. Estimated bounds on the resistance of the pipe 17_18; $L=0$.

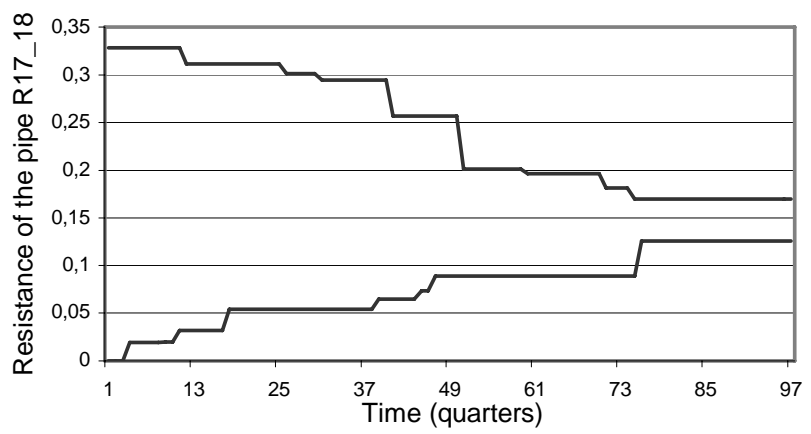

Fig. 4. Estimated bounds on the resistance of the pipe 17_18; $L=2$.

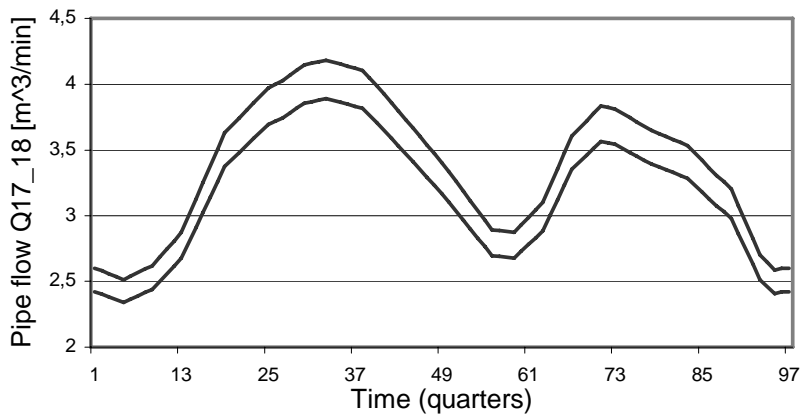

Fig. 5. Estimated bounds on the pipe flow Q17_18; $L=0$.

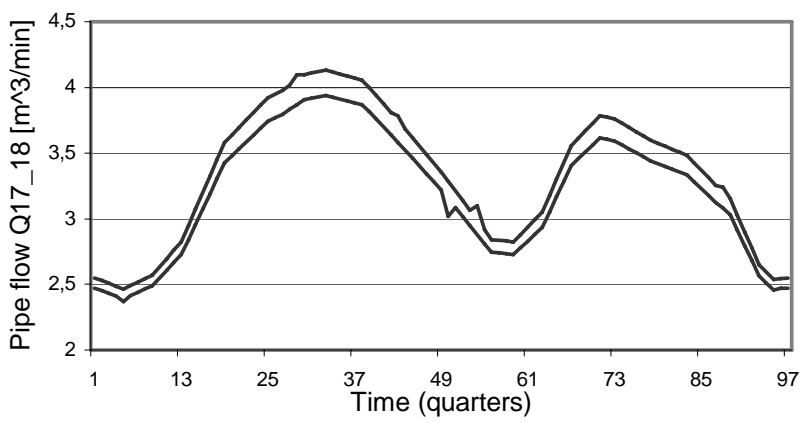

Fig. 6. Estimated bounds on the pipe flow Q17_18; $L=2$.

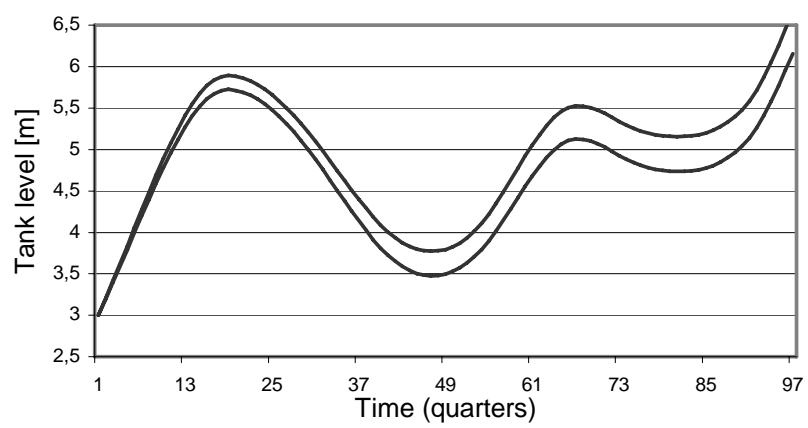

Fig. 7. Estimated bounds on the tank level; $L=0$.

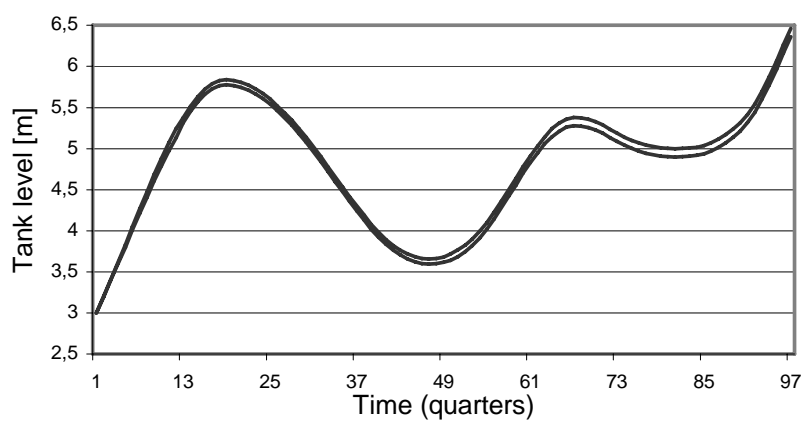

Fig. 8. Estimated bounds on the tank level; $L=2$.

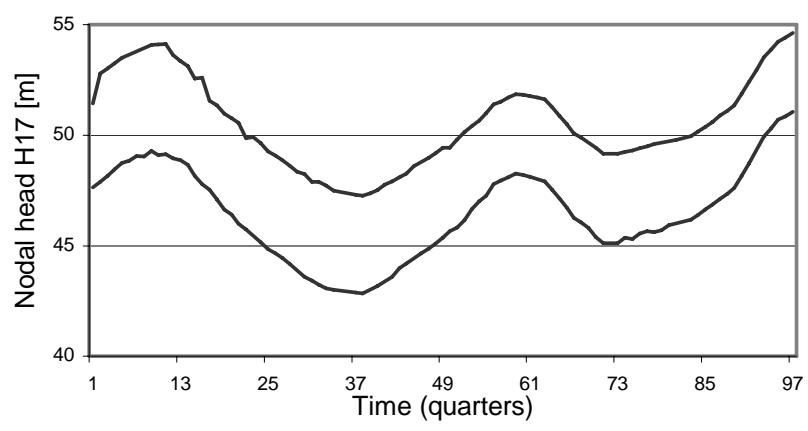

Fig. 9. Estimated bounds on the nodal head H17; $L=0$.

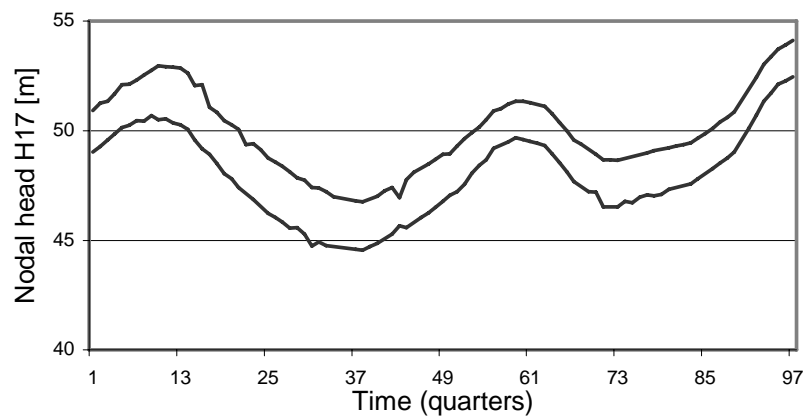

Fig. 10. Estimated bounds on the nodal head H17; $L=2$. 


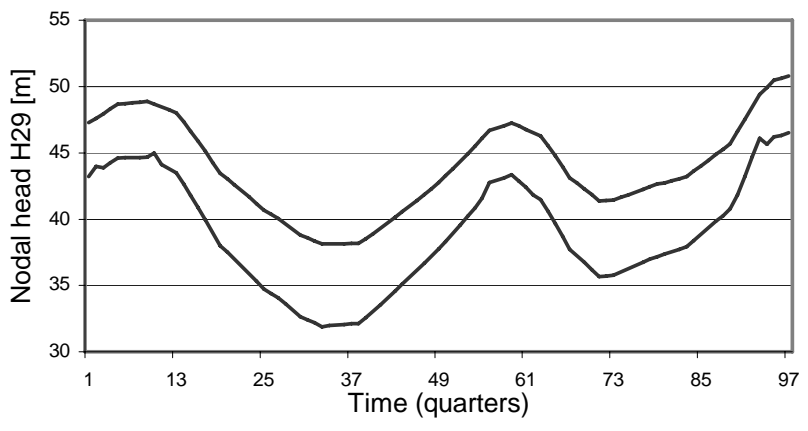

Fig. 11. Estimated bounds on the nodal head $\mathrm{H} 29 ; L=0$.

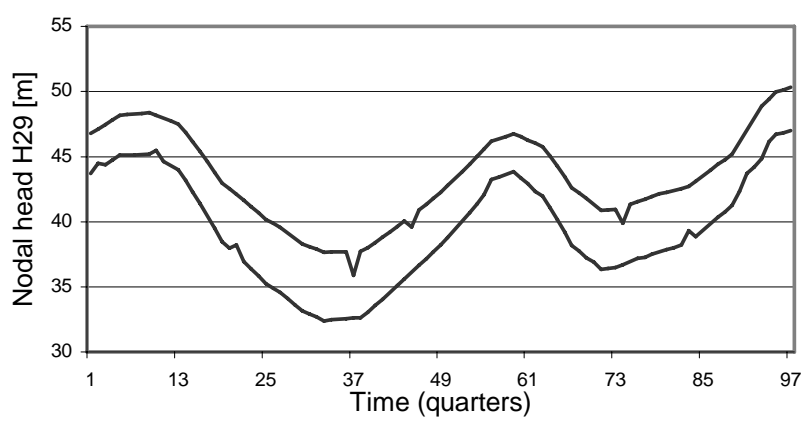

Fig. 12. Estimated bounds on the nodal head $\mathrm{H} 29 ; L=2$.

\section{Conclusions}

The paper considered a set membership joint estimation of variables and parameters in dynamic networks. A recursive estimation algorithm suitable for on-line network monitoring was derived. It was validated on a case-study water distribution network. The results showed significant improvements of the estimates produced by estimation with moving measurement window. An analysis of rigorous criteria for the selection of the window length in order to reach a desired trade-off between estimation accuracy and computing effort are under research. The application of the recursive algorithm with a moving measurement window to a joint estimation of integrated quality and quantity in complex drinking water distribution networks is described in (Duzinkiewicz, 2005).

\section{Acknowledgment}

This work has been supported by the Ministry of Science and Higher Education under the grant no. 4 T11A 00825. The authors wish to express their thanks for the support.

\section{References}

Brdyś M.A. and Chen K. (1996): Joint estimation of states and parameters of integrated quantity and quality models of dynamic water supply and distribution systems. - Proc. 13-th IFAC World Congress, San Francisco, CA, Vol. 1, pp. 73-78, .

Brdyś M.A. and Lisiak Z. (1999): Robust soft sensors for on-line monitoring of mixing quality in water supply networks. Proc. European Control Conf., ECC'99, Karlsruhe, Germany, (CD-ROM).

Brdyś M.A., Duzinkiewicz K., Grochowski M. and Rutkowski T. (2001): Robust estimation of integrated hydraulics and parameters in water distribution systems. - Proc. 4-th ASCE Annual Water Distribution Systems Analysis, 2001 World Water and Environmental Resources Congress, Orlando, FL, (CD-ROM).

Chang T., Duzinkiewicz K. and Brdyś M.A. (2004): Bounding approach to parameter estimation of point-parametric models. - Proc. 10-th IFAC/IFORS/IMACS/IFIP Symp. Large Scale Complex Systems, Osaka, Japan, Vol. 1, pp. 233-238.

Duzinkiewicz K. (2005): Integrated control of drinking water supply and distribution systems. - D.Sc. thesis, AGH University of Science and Technology Publishers, Cracow, (in Polish).

Milanese M., Norton J.P., Piet-Lahanier H. and Walter E. (1996): Bounding Approaches to System Identification. - New York: Plenum Press.

Raïssi T., Ramdani N. and Candau Y. (2004): Set membership state and parameter estimation for systems described by nonlinear differential equations. - Automatica, Vol. 40, No. 10, pp. 1771-1777.

Rutkowski T., Brdyś M.A., Konarczak K. and Gmiński T. (2004): Set-bounded joined parameter and state estimation for model predictive control of integrated wastewater treatment plant systems at medium time scale. - Proc. 10th IFAC/IFORS/IMACS/IFIP Symp. Large Scale Complex Systems, Osaka, Japan, Vol. 2, pp. 789-794.

Schweppe F.C. (1978): Uncertain Dynamic Systems. — Englewood Cliffs, New Jersey: Prentice Hall.

Veres S.M. and Norton J.P. (1991): Structure selection for bounded-parameter models: Consistency conditions and selection criterion. - IEEE Trans. Automat. Contr., Vol. 36, No. 4, pp. 474-481.

Walter E. and Pronzato L. (1997): Identification of Parametric Models from Empirical Data. — Berlin: Springer.

Received: 12 October 2005 Revised: 20 March 2006 